\title{
Genome-wide identification of specific oligonucleotides using artificial neural network and computational genomic analysis Chun-Chi Liu'1,2,3, Chin-Chung Lin'², ${ }^{2}$ Ker-Chau Li ${ }^{3}$, Wen-Shyen E Chen ${ }^{1}$, Jiun-Ching Chen ${ }^{4}$, Ming-Te Yang ${ }^{4}$, Pan-Chyr Yang ${ }^{5}$, Pei-Chun Chang ${ }^{\dagger 6}$ and Jeremy JW Chen*+2,4,5
}

\begin{abstract}
Address: ${ }^{1}$ Department of Computer Science, National Chung-Hsing University, Taichung, Taiwan, ROC, ${ }^{2}$ Institute of Biomedical Sciences, National Chung-Hsing University, Taichung, Taiwan, ROC, ${ }^{3}$ Institute of Statistical Science, Academia Sinica, Taipei, Taiwan, ROC, ${ }^{4}$ Institute of Molecular Biology, National Chung-Hsing University, Taichung, Taiwan, ROC, ${ }^{5}$ NTU Center for Genomic Medicine, National Taiwan University

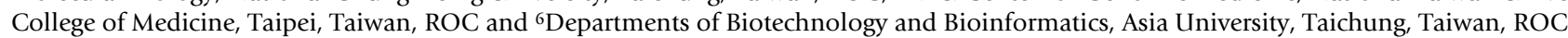

Email: Chun-Chi Liu - jjliu@cs.nchu.edu.tw; Chin-Chung Lin - casera@gmail.com; Ker-Chau Li - kcli@stat.sinica.edu.tw; WenShyen E Chen - echen@cs.nchu.edu.tw; Jiun-Ching Chen - imjaco@msn.com; Ming-Te Yang - mtyang@dragon.nchu.edu.tw; PanChyr Yang - pcyang@ha.mc.ntu.edu.tw; Pei-Chun Chang - pcchang@asia.edu.tw; Jeremy JW Chen* - jwchen@dragon.nchu.edu.tw

* Corresponding author †Equal contributors
\end{abstract}

Published: 22 May 2007

BMC Bioinformatics 2007, 8:164 doi:10.1/86/1471-2105-8-164
Received: 24 October 2006

Accepted: 22 May 2007

This article is available from: http://www.biomedcentral.com/147I-2/05/8/164

(c) 2007 Liu et al; licensee BioMed Central Ltd.

This is an Open Access article distributed under the terms of the Creative Commons Attribution License (http://creativecommons.org/licenses/by/2.0), which permits unrestricted use, distribution, and reproduction in any medium, provided the original work is properly cited.

\begin{abstract}
Background: Genome-wide identification of specific oligonucleotides (oligos) is a computationallyintensive task and is a requirement for designing microarray probes, primers, and siRNAs. An artificial neural network (ANN) is a machine learning technique that can effectively process complex and high noise data. Here, ANNs are applied to process the unique subsequence distribution for prediction of specific oligos.

Results: We present a novel and efficient algorithm, named the integration of ANN and BLAST (IAB) algorithm, to identify specific oligos. We establish the unique marker database for human and rat gene index databases using the hash table algorithm. We then create the input vectors, via the unique marker database, to train and test the ANN. The trained ANN predicted the specific oligos with high efficiency, and these oligos were subsequently verified by BLAST. To improve the prediction performance, the ANN over-fitting issue was avoided by early stopping with the best observed error and a k-fold validation was also applied. The performance of the IAB algorithm was about 5.2, 7.I, and 6.7 times faster than the BLAST search without ANN for experimental results of 70-mer, 50-mer, and 25-mer specific oligos, respectively. In addition, the results of polymerase chain reactions showed that the primers predicted by the IAB algorithm could specifically amplify the corresponding genes. The IAB algorithm has been integrated into a previously published comprehensive web server to support microarray analysis and genome-wide iterative enrichment analysis, through which users can identify a group of desired genes and then discover the specific oligos of these genes.

Conclusion: The IAB algorithm has been developed to construct SpecificDB, a web server that provides a specific and valid oligo database of the probe, siRNA, and primer design for the human genome. We also demonstrate the ability of the IAB algorithm to predict specific oligos through polymerase chain reaction experiments. SpecificDB provides comprehensive information and a user-friendly interface.
\end{abstract}




\section{Background}

DNA microarray is a powerful tool in functional genome studies [1-4]. However, it usually generates false positive data as a result of cross-hybridization between highly similar sequences [5-7]. The design approach of polymerase chain reaction (PCR) primer with minimal cross homology is an important technology [8]. In addition, the recent application of siRNAs to silence genes is dependent on the sequence specificity, and the siRNA sequence must be selected carefully to avoid similarity to an unrelated mRNA [9]. Thus, the important issue is finding a way to effectively identify specific oligonucleotides (oligos).

The early design of specific oligos was based mainly on the use of a frequency matrix $[10,11]$. Subsequently, several approaches were developed to design unique oligos, such as an information-theoretical method based on maximum entropy, which has also been applied to the design of probe sets [12]; a method based on matching the frequency of sequence landscapes, which was used to select optimal oligos for E. coli, S. cerevisiae, and C. elegans [13]; suffix trees, which has been used to select the organismspecific signature oligos [14]; the design of genome-wide specific oligos based on basic local alignment search tool (BLAST) [15]; and the smart filtering technique, which was employed to avoid redundant computation while maintaining accuracy [16].

However, these processes still take a long time to identify specific oligos. It is quite obvious that the high-throughput prediction of specific oligos is important for application in large-scale gene analysis. Recently, a method for unique oligo discovery that was a modification of a central pattern partitioning principle was published [17]. This method analyzed 17 complete genomes representing a wide range of both prokaryotic and eukaryotic organisms. However, huge genomes, such as the human genome, were not processed in this report.

An artificial neural network (ANN) is a popular learning approach that effectively handles noise and complex relationships in a robust way [18]. In previous studies, ANNs were employed to process a broad range of input parameters on sequence information, such as base composition and binding properties, to predict anti-sense oligos targeting the mRNA $[19,20]$. In addition, ANNs have been widely applied to various research fields in biology such as clinical cancer research [21], protein function prediction [22], protein classification [23], and cancer classification [24].

In this study, we present a novel and efficient algorithm that integrates ANN and BLAST, named the IAB algorithm, to identify specific oligos from the Institute for Genomic Research (TIGR) human gene index (HGI) and rat gene index (RGI) databases. Furthermore, we applied the IAB algorithm to construct SpecificDB, a web server that provides users with the appropriate hybridization probe, siRNA, and primer for the HGI sequences. These tools will be of great benefit to functional genomics studies.

\section{Results \\ Construction of unique marker database and the architecture of ANN}

The input vector of the ANN was derived from the density of the unique subsequences $\left(U_{d}\right)$ between 10-mer and 26mer (Figure 1). In our previous study, an algorithm with 15-mer $U_{d}$ was developed to speed up the identification of a specific probe [1] and we extended and enhanced the algorithm in this study. We established a unique marker database (UMD) to store the positions of all unique subsequences for the entire TIGR HGI tentative human consensus (THC) database and RGI tentative consensus (TC) database [25]. The UMD included of 10-mer 26-mer unique marker subsequences and its workflow is illustrated in Figure 2. Determining the appropriate weights of 10-mer $\sim 26$-mer $U_{d}$ for the specific oligo prediction was a difficult issue, and the optimal weights depended on the sequence constitution of the genome.

In this study, to determine the optimal weights of 10-mer $\sim 26$-mer $U_{d}$, ANN was utilized in our algorithm to predict the oligo specificity. Table 1 shows the number of unique markers and the average density of 10-mer 26-mer unique subsequences in the UMD of HGI and RGI. The results reveal that if the length of the screening subsequence ( $\mathrm{N}$-mer) was less than 12-mer, most subsequences were not unique in a large database $\left(U_{d}\right.$ approximates to $0)$. On the other hand, if the N-mer was more than 24mer, many subsequences would be unique $\left(U_{d}\right.$ of HGI approximates to 0.23 and $U_{d}$ of RGI approximates to $0.49)$. Therefore, the construction of the unique marker subsequences with 10-mer 26-mer in the UMD was reasonable. The architecture of ANN's backward propagation is shown in Figure 1b. There were 17 input nodes in the ANN for the $U_{d}$ of 10-mer $\sim 26$-mer. The cross homology calculated by WU-BLAST [26] for each input sequence was as the desired output.

\section{Over-fitting and validation}

If too much training is applied to the training set, over-fitting of the ANN will occur, which means that it will be fitted precisely to the training set and thereby lose accuracy in the independent test set. Over-fitting would be expected with sufficiently large ANNs and sufficiently "successful" training [27]. The results of over-fitting tests in this study revealed that the over-fitting effect was dependent on the number of hidden layer nodes (Figure 3). 

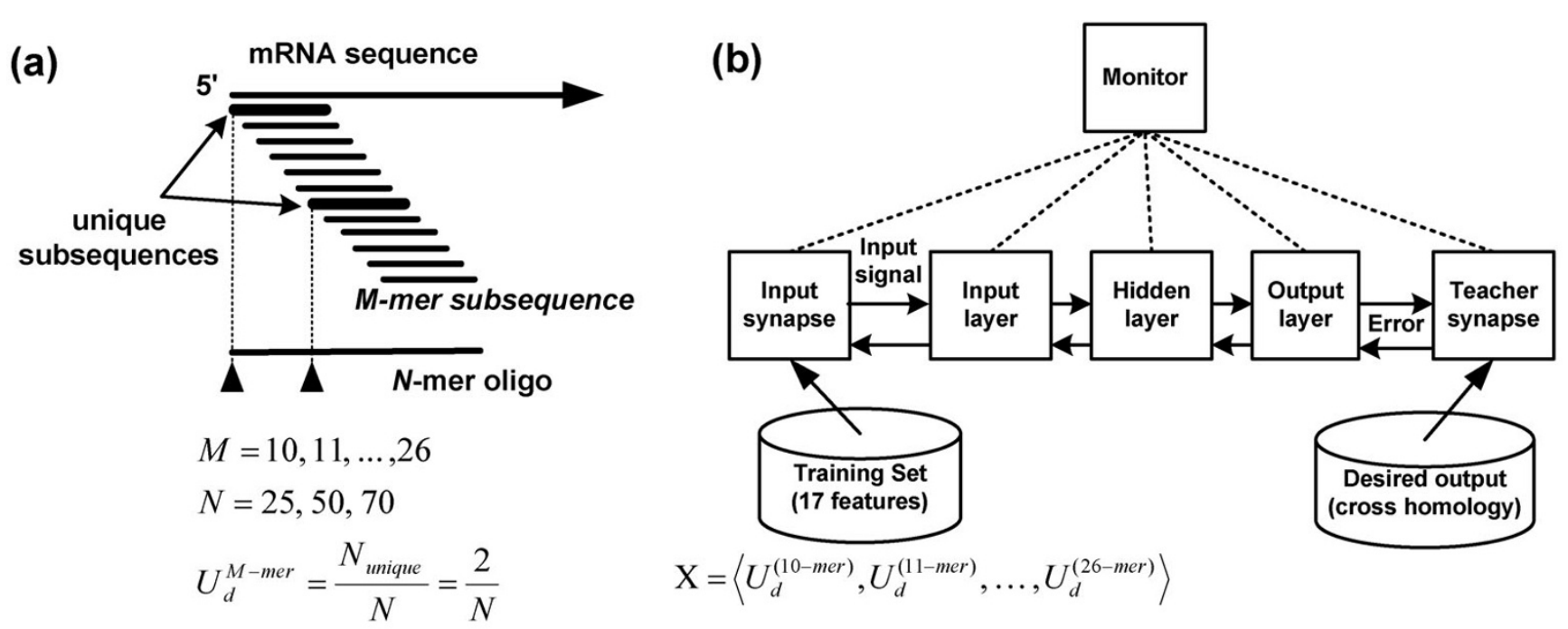

(c)

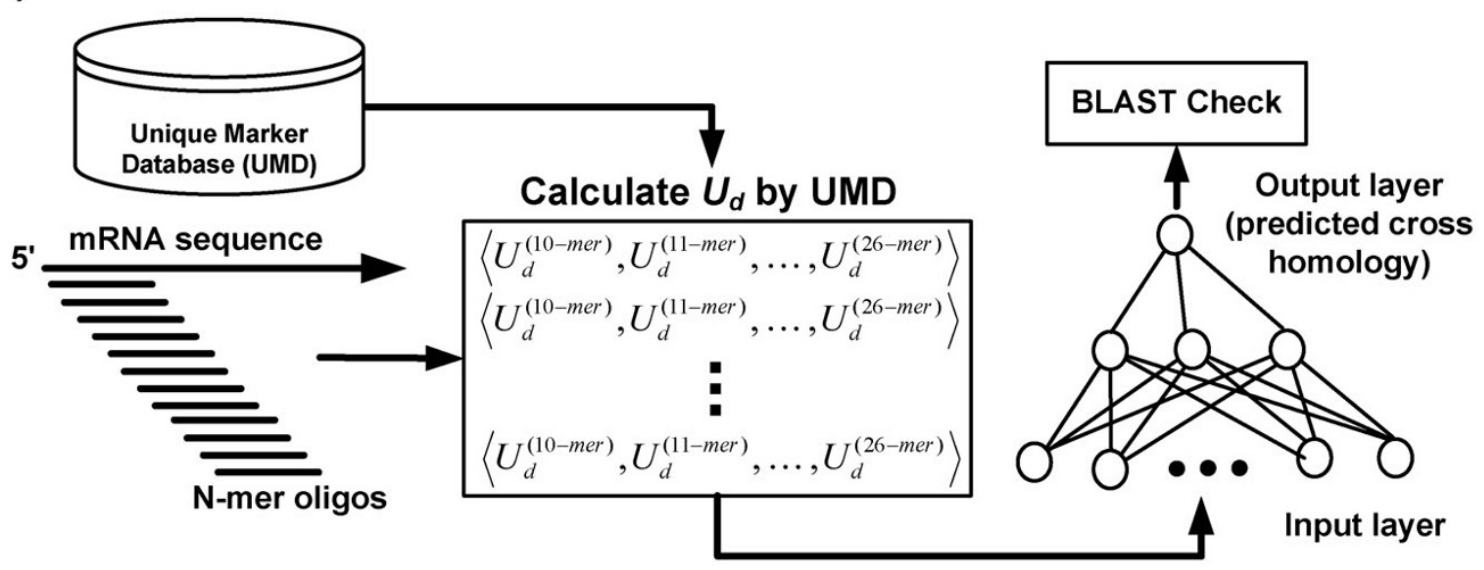

Figure I

Integration of ANN and BLAST (IAB). (a) Calculation of the density of unique subsequence: the solid triangles mark the starting position of the unique subsequences in an $\mathrm{N}$-mer oligo, and the count of the solid triangles is the number of unique subsequences. Thus, the $M-$ mer $U_{d}$ can be calculated from the number of unique subsequences. (b) ANN training: there were I 7 input nodes in the ANN for the input vector ( 10 -mer 26-mer $\left.U_{d}\right)$ that is calculated in (a). In addition, the cross homology identified by WU-BLAST was as the desired output. The monitor object represents the central point that contains all of the parameters needed for other components to work properly. (c) IAB algorithm architecture: for each sliding N-mer oligo, the input vector $\left(10\right.$-mer 26-mer $\left.U_{d}\right)$ calculated by the unique maker database (UMD) was delivered to the ANN for cross homology prediction. The selected oligos were checked by BLAST after filtering by ANN scores.

Our results show that the over-fitting effect of the ANN performance with 22 hidden nodes (Figure $3 \mathrm{~b}$ ) is more pronounced than that with 16 hidden nodes (Figure 3a). Furthermore, root mean square error (RMSE) [28] became quite stable and relatively lower when the training cycle number was between 350 and 500. The best observed error for the test set happened when the training cycle was 450 (RMSE $=0.0767$, Figure 3b). Thus, the setting of 450 cycles was applied to all future procedures. Furthermore, k-fold validation was performed on nine training sets [29]. Thus, nine trained ANNs were produced and tested using the independent test set. In addition, we also evaluated the ANN's performance using an independent largescale validation set. The results show that the RMSEs of the test and validation sets had similar profiles and the best RMSE occurred in the same training set $\left(\mathrm{TS}_{\mathrm{THC} 186}\right)$ for both the test and validation sets (Figure 4). The consistent profile of both the test and validation sets indicates the 


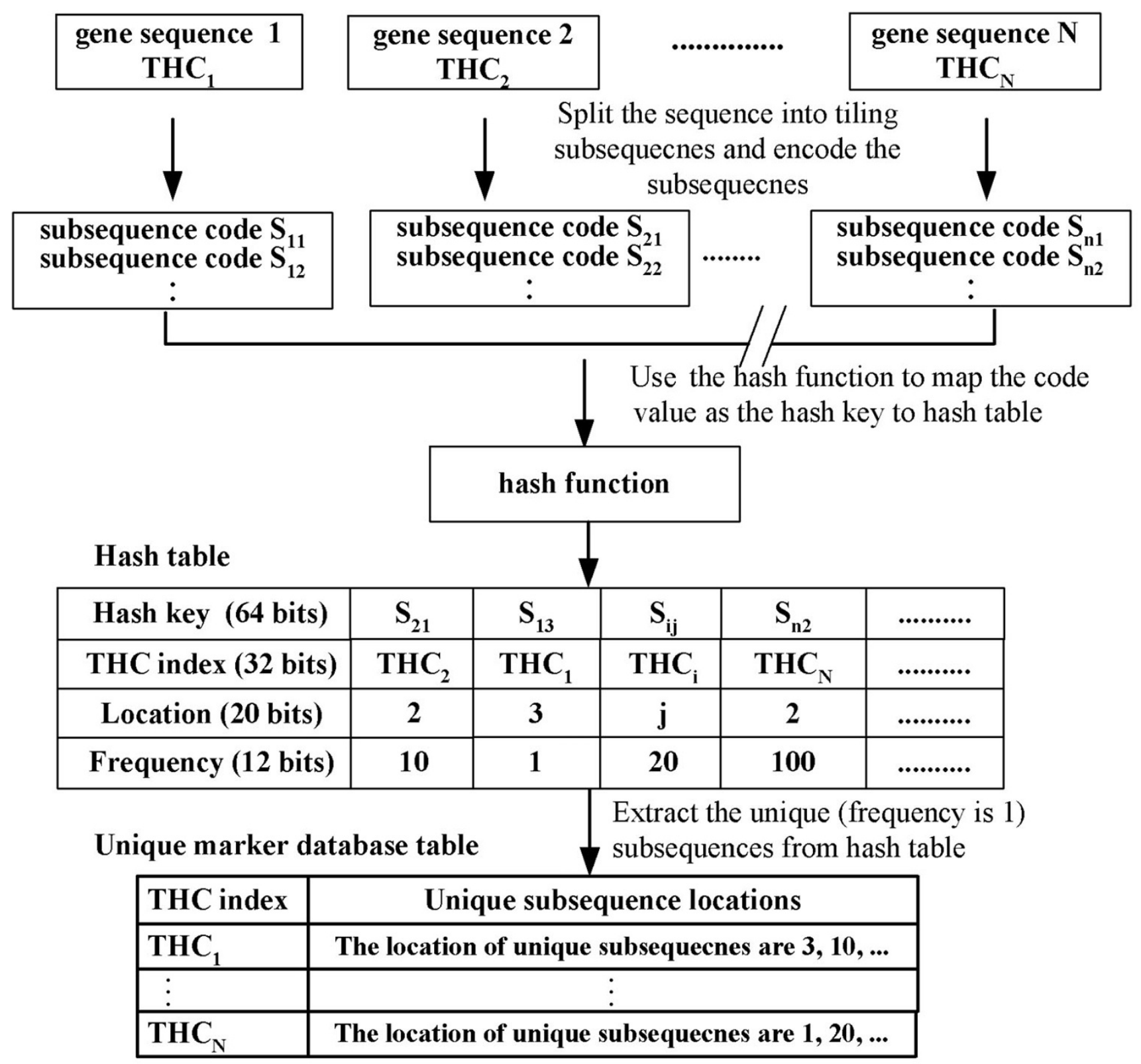

Figure 2

A flowchart depicting the procedures for the creation of a UMD. The subsequences and their complementary sequences for all of the genes in the database were encoded and placed in the hash table using the coding number of the subsequences as the hash key. If the subsequence appears only once, the subsequence is treated as unique, and then its location will be stored in the marker tables of UMD.

stability of the ANN's performance. Thus, the ANN trained by $\mathrm{TS}_{\mathrm{THC186}}$ was selected for genome-wide identification of the specific oligos.

\section{Performance comparison for IAB algorithm}

To investigate the performance at the various lengths of oligos, 100 THC sequences on which to perform the IAB algorithm, the pure BLAST search, and the BLAST search with $U_{d}$ were randomly selected from the HGI database. Three performance criteria were computed and evaluated, including success rate, average cross homology and execution time. The sensitivity factor is the maximum percentage of sliding oligos that should be screened by BLAST. Thus, the sensitivity factor is used to adjust the sensitivity of the IAB algorithm in this report. Moreover, to investigate the effect of the sensitivity factor on performance, var- 
Table I: The number of unique markers and the average density of I0-mer $\sim 26-$ mer subsequences in the database of HGI and RGI.

\begin{tabular}{|c|c|c|c|c|}
\hline \multirow[t]{2}{*}{$N$-mer } & \multicolumn{2}{|c|}{$H G l^{a}$} & \multicolumn{2}{|c|}{$R G I^{b}$} \\
\hline & Unique Markers & Average $U_{d}^{c}$ & Unique Markers & Average $U_{d}$ \\
\hline 10-mer & 0 & 0.000000 & 4 & 0.000000 \\
\hline II-mer & 6 & 0.000000 & 9,669 & 0.000120 \\
\hline 12-mer & 24,164 & 0.000054 & 457,681 & 0.005675 \\
\hline 13-mer & $|| 24,,49 \mid$ & 0.002513 & $4,167,009$ & 0.051672 \\
\hline 14-mer & $9,488,257$ & 0.021202 & $15,364,798$ & 0.190527 \\
\hline 15-mer & $35,768,666$ & 0.079925 & $27,962,463$ & 0.346742 \\
\hline 16-mer & $66,958,259$ & 0.149618 & $34,903,375$ & 0.432811 \\
\hline 17-mer & $85,694,125$ & 0.191484 & $37,636,118$ & 0.466698 \\
\hline 18-mer & $94,052,393$ & 0.210160 & $38,645,904$ & 0.479219 \\
\hline 19-mer & $97,689,916$ & 0.218288 & $39,061,604$ & 0.484374 \\
\hline 20-mer & $99,559,386$ & 0.222466 & $39,276,816$ & 0.487043 \\
\hline $21-\mathrm{mer}$ & $100,781,233$ & 0.225196 & $39,4|7,68|$ & 0.488789 \\
\hline 22-mer & $101,743,85 \mid$ & 0.227347 & $39,522,956$ & 0.490095 \\
\hline 23-mer & $102,583,079$ & 0.229222 & $39,608,919$ & 0.491161 \\
\hline 24-mer & $103,348,039$ & 0.230931 & $39,682,141$ & 0.492069 \\
\hline 25-mer & $104,057,929$ & 0.232518 & $39,744,826$ & 0.492846 \\
\hline 26-mer & $104,724,128$ & 0.234006 & $39,800,164$ & 0.493532 \\
\hline
\end{tabular}

aHGI, human gene index; ${ }^{b} R G I$, rat gene index; ${ }^{c} U_{d}$, the density of the unique subsequences.

ious sensitivity factors were evaluated. The results reveal that the IAB algorithm relative to other approaches had better or equal quality with a sensitivity factor of 0.3 for 70-mer, 50-mer, and 25-mer in the success rate, average cross homology, and execution time (see Table 2). Thus, the sensitivity factor was set at 0.3 in all performance comparisons.

In HGI database, the IAB algorithm was executed about $5.2,7.1$, and 6.7 times faster than the pure BLAST search for 70-mer, 50-mer, and 25-mer, respectively (Table 2). In the comparison of the BLAST search with $U_{d}$, the IAB algorithm performed about 3.0, 2.3, and 3.0 times faster for 70-mer, 50-mer, and 25-mer, respectively. In 25-mer specific oligo design, Table 2 shows that the IAB algorithm decreased execution times by 6.2, 7.0, and 7.0 times for word lengths of 11,8 , and 5, respectively. In RGI database, the IAB algorithm, the pure BLAST search, and the BLAST with $U_{d}$ for 70-mer specific oligos were performed. The IAB algorithm was executed about 7.3 times faster than the pure BLAST search and 2.0 times faster than the BLAST with $U_{d}$ (Table 3 ). In addition, the BLAST with $U_{d}$ was executed about 3.6 times faster than the pure BLAST search. It is expectable that the BLAST with $U_{d}$ has better performance than the pure BLAST search and the IAB algorithm has better performance than the BLAST with $U_{d}$ algorithm.

\section{Specific oligo web server (SpecificDB)}

To provide a useful and powerful web server named SpecificDB, the following steps were taken: (1) unique subsequences with 10-mer 26-mer were created in UMD; (2) training, test, and validation sets were prepared; (3) k-fold validation was performed on training sets; $(4)$ the IAB algorithm was implemented; (5) specific oligos including probe, siRNA, and primer were constructed; and (6) the specific oligo database was incorporated into our previous work (a comprehensive web server for the composite regulatory signature database, CRSD) [30] consisting of microarray analysis, motif discovery, and genome-wide iterative enrichment analysis for microRNAs, transcription factors, pathways, and GO annotations. Users can perform microarray data analysis and enrichment analysis to identify a group of interesting genes, and then discover the specific oligos for the probe, siRNA, and primer of these genes in the SpecificDB web server. The architecture of SpecificDB is illustrated in Figure 5, and the server is available at our web site [31].

\section{Application of IAB algorithm and the demonstration of predicted primers}

The IAB algorithm was applied to identify the specific primers of Xanthomonas campestris pv. Campestris (Xcc) strain 17 contigs that were constructed and sequenced. To locate all of the genes in Xcc strain 17, another similar strain, $X c c$ strain 33913 containing 4,181 genes [32], was employed to perform sequence alignment. The required information and annotation of Xcc strain 33913 genome is available in the NCBI database.

We aligned the 4,181 gene sequences against the Xcc strain 17 contigs to obtain the annotation data of the contigs. The results showed that the average sequence similarity between the two strains was $94.81 \%$. There were 3,836 genes with similarity in excess of $90 \%$ that were selected as the predicted genes in $X c c$ strain 17. In order to avoid the non-specific annealing of predicted primers, three 
(a)

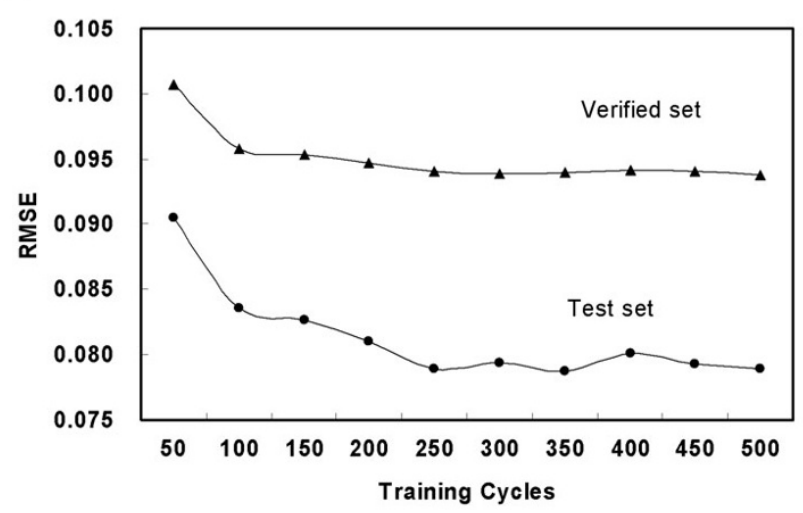

(b)

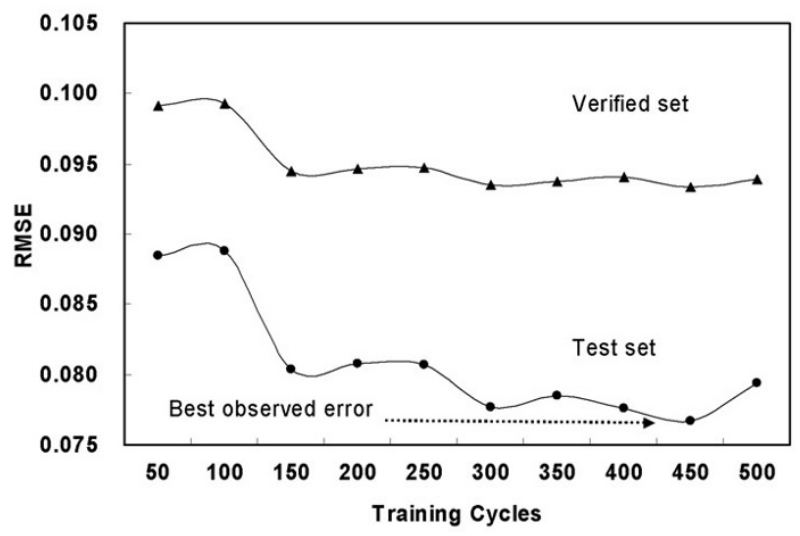

Figure 3

The performance profiles with different training epochs for test and validation sets. The ANNs were trained with different numbers of hidden layer nodes as follows: (a) 16 hidden nodes, and (b) 22 hidden nodes. The profiles show that the optimal performance for the test and validation sets did not occur at the maximum training epochs. RMSE became more stable and relatively lower when the training epoch number was between 350 and 500 . The best observed error for the test set is RMSE 0.0767 at 450 (b).

additional genomes (A. thaliana, S. cerevisiae, and E. coli) were merged with the Xcc strain 17 contigs to build an integrated nucleotide sequence database that was used for non-specific oligo filtration. A total of 3,569 primer sets were identified from 3,836 genes by using a cross homology threshold of $85 \%$ against the integrated nucleotide sequence database [see Additional file 1]. More than 93\% of all the genes contained the specific and valid primer sets.

To demonstrate the applicability of predicted primers, we selected 18 and 29 genes related to SOS response [33] and rpoE [34] respectively, as well as 49 randomly selected genes in Xcc strain 17 to perform PCR amplification using the primers predicted by the IAB algorithm. In addition, to verify the primer design based on the IAB algorithm for large genomes such as human, we randomly selected 15 human primer sets from our SpecificDB database and performed PCR amplification. A total of 111 PCR reactions (96 for Xcc and 15 for human) were performed in twice and followed by electrophoresis analysis. If a PCR product with correct size can be found at least once, we count the result as a success. The PCR results show that the success rate was 95\% and 93\% for Xcc and human, respectively, and all PCR products had correct size. Representative results are shown in Figure 6. In human PCR results, there is one gene (NM 052957) that has multiple bands but a single dominant band with correct size.

\section{Discussion}

Several important techniques in molecular biology, such as siRNA, microarray, and primer design, need short and specific oligos. The prediction of short and specific oligos is essential for these applications $[9,35]$. The BLAST search can be utilized to deal with the identification of specific oligos [36] but it requires too much computing time to screen all sliding oligos. Thus, a fast and efficient predictor for sequence specificity is needed [37]. In this study, ANN is used as a predictor to filter out the oligos with high cross homology before the BLAST search. Here, a new method for genome-wide identification of specific oligos is developed, and it integates ANN and BLAST to optimize the sequence analysis by using the densities of the various length unique subsequences.

Early in this study, we investigated repeat frequencies of subsequence (6-mer 12-mer) and Shannon's entropy of subsequence frequencies distributions [38], which are related to the degeneracy of the subsequence coding scheme. However, we did not discover an efficient method to integrate these distributions (unpublished data). In this report, we found that the integration of the 10-mer $\sim 26$-mer $U_{d}$ and ANN is an efficient approach to predict oligo specificity.

In siRNA design, BLAST is frequently used to determine the specificity of siRNAs. However, BLAST may lose sensitivity and miss important alignment for such short oligos as siRNAs $[39,40]$. On the other hand, the sensitivity of BLAST depends on the word length parameter so that shorter word lengths may increase sensitivity but decrease execution speed. To investigate the impact of the word length, several word lengths $(11,8$, and 5$)$ were employed to evaluate the performance of our algorithm on specific short oligo identification. Table 2 shows that the IAB algorithm enjoys significant improvement in speed for various word lengths. Thus, the shorter word length can be applied to improve sensitivity in the IAB algorithm. 


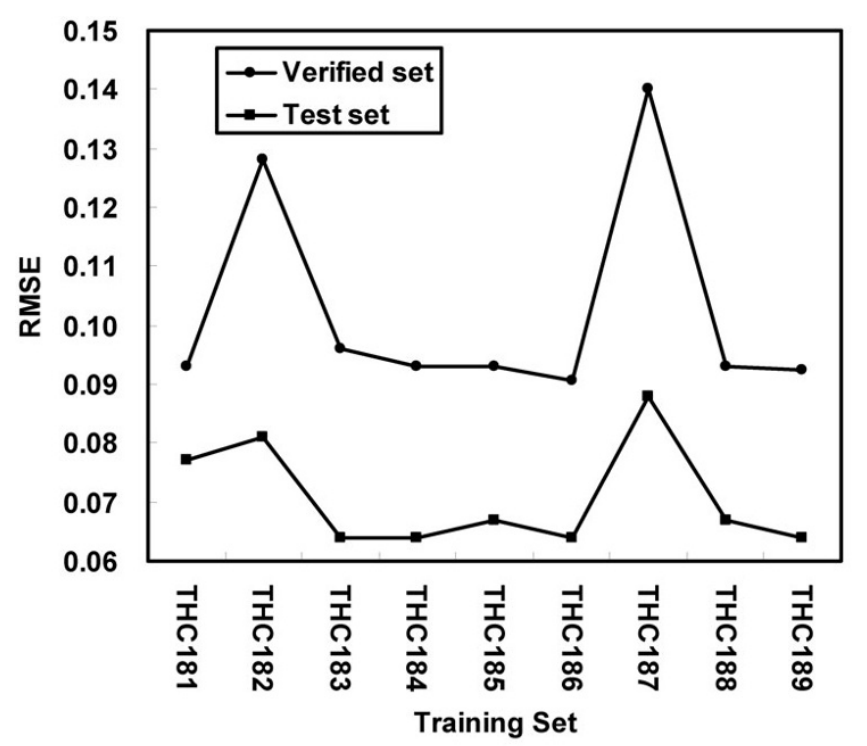

Figure 4

The performance profiles of a k-fold validation. The RMSEs of the test and validation sets have the same trend, which signals the generality of the result. The best performance occurred in training set $\mathrm{TS}_{\mathrm{THCl} 86}$ for both the test and validation sets.

The combination of ANN and $U_{d}$ is a part of the IAB algorithm. The performance comparison of the IAB algorithm and the BLAST search with $U_{d}$ revealed that ANN is an important component in the IAB algorithm (Table 2). The IAB algorithm with appropriate sensitivity factor had lower cross homology and shorter execution time. Although the IAB algorithm only screened a portion of the sliding oligos, it still had better quality than the pure BLAST search that may screen all sliding oligos.

It is difficult to understand the inside workings of an ANN, where learned knowledge is contained in the weight (coefficient) of synapse in the ANN structure. Thus, the ANN is usually treated as a black box [41] and the biological significance inside can not be interpreted. However, ANNs have been applied to various research fields in bioinformatics. In addition, the unique subsequence distribution has also been utilized in various aspects of sequence analysis $[1,42]$. In this study, we integrated a wide range of unique subsequences (10-mer $~ 26$-mer) using the ANN approach to improve the identification of specific oligos. Such a wide range of unique subsequences has not been previously reported.

ANN training for every genome and every length of oligo is inflexible and inconvenient for applications, but our findings indicate that the $\mathrm{IAB}$ algorithm may overcome this problem. Table 2 demonstrates the robustness of our algorithm by applying the ANN trained for 70-mer oligos to the prediction of 50-mer and 25-mer specific oligos. Our results show that the predictions for the 50-mer and 25-mer specific oligos have similar performance to that of the 70-mer.

Furthermore, to understand whether the final trained ANN from HGI could be applied to other genomes, we derived the 70-mer training, test, and validation sets from RGI, and performed a k-fold validation method to obtain the best trained ANN. Then, we randomly selected 200 RGI TC sequences to carry out the specific oligo selection by using the trained ANN of HGI and RGI with IAB, as well as the pure BLAST search and the BLAST with $U_{d}$. The results of the experiments show that trained ANN from both HGI and RGI had almost the same performance in specific oligo selection for the RGI database (Table 3). Therefore, it may be not necessary to perform the complicated procedures of training a new ANN for RGI. Thus, the final trained ANN from HGI has robustness to other genomes such as RGI, and the IAB algorithm can be employed across species for specific oligo identification.

Biological researchers may obtain a list of marker genes related to human diseases or the gene expression signature derived from microarray analysis. In order to provide these researchers with useful bioinformatic tools to further investigate the genes, our SpecificDB web server provides a web interface to perform microarray data analysis and discover significant enrichment of microRNAs, transcription factors, pathways, and GOs. For example, after enrichment analysis, users can identify a panel of genes that may have significant differential expression in microarray data and have significant enrichment with a pathway. SpecificDB can bring out the specific and valid probes, siRNAs, and primers corresponding to these genes.

To demonstrate the applicability of our algorithm, Xcc genome analysis and genome-wide primer design have been carried out. S. cerevisiae and E. coli are usually the major contaminants in the laboratory environment, which may influence the accuracy of experiments $[43,44]$. To reduce the cross homology with these species, we established an integrated nucleotide sequence database consisting of four genomes (A. thaliana, S. cerevisiae, E. coli, and $X c c$ ) for non-specific oligo filtration. Nevertheless, the results of this primer design can not be treated as completely species-specific primers. The results of PCR amplification with primers predicted by the IAB algorithm provide evidence in support of the effectiveness and accuracy of our novel algorithm.

\section{Conclusion}

A new algorithm, the IAB algorithm, integrates ANN and BLAST to select specific oligos, and makes use of the 
Table 2: Performance comparison with and without an artificial neural network.

\begin{tabular}{|c|c|c|c|c|c|}
\hline Oligo length & Procedure & Sensitivity factor & Success rate $(\%)^{\mathrm{a}}$ & Cross homology b & Execution time (hours) \\
\hline \multirow[t]{5}{*}{ 70-mer } & $\mid A B B^{c}$ & 0.2 & 94 & 0.56 & 0.50 \\
\hline & & 0.3 & 95 & 0.56 & 0.71 \\
\hline & & 0.4 & 95 & 0.56 & 0.90 \\
\hline & $\mathrm{BL}$ & with $U_{d}{ }^{d}$ & 95 & 0.56 & 2.13 \\
\hline & & BLAST & 95 & 0.61 & 3.69 \\
\hline \multirow[t]{5}{*}{ 50-mer } & $I A B$ & 0.2 & 91 & 0.64 & 0.20 \\
\hline & & 0.3 & 93 & 0.64 & 0.31 \\
\hline & & 0.4 & 93 & 0.64 & 0.32 \\
\hline & & with $U_{d}$ & 93 & 0.64 & 0.70 \\
\hline & & BLAST & 93 & 0.69 & 2.19 \\
\hline \multirow[t]{5}{*}{$25-\operatorname{mer} W=11 \mathrm{e}$} & $I A B$ & 0.2 & 94 & 0.79 & 0.19 \\
\hline & & 0.3 & 94 & 0.79 & 0.26 \\
\hline & & 0.4 & 94 & 0.79 & 0.33 \\
\hline & & with $U_{d}$ & 94 & 0.79 & 0.78 \\
\hline & & BLAST & 94 & 0.80 & 1.62 \\
\hline \multirow[t]{5}{*}{$25-$ mer W = 8} & $\mathrm{IAB}$ & 0.2 & 93 & 0.80 & 0.36 \\
\hline & & 0.3 & 93 & 0.80 & 0.50 \\
\hline & & 0.4 & 93 & 0.80 & 0.64 \\
\hline & & with $U_{d}$ & 93 & 0.80 & 1.52 \\
\hline & & BLAST & 93 & 0.81 & 3.51 \\
\hline \multirow[t]{5}{*}{$25-\operatorname{mer} W=5$} & $I A B$ & 0.2 & 92 & 0.81 & 1.00 \\
\hline & & 0.3 & 92 & 0.81 & 1.39 \\
\hline & & 0.4 & 92 & 0.81 & 1.79 \\
\hline & & with $U_{d}$ & 92 & 0.81 & 4.25 \\
\hline & & BLAST & 92 & 0.81 & 9.69 \\
\hline
\end{tabular}

aThe success rate is the percentage of tentative human consensus sequences on which the procedure can find the specific oligo (where the cross

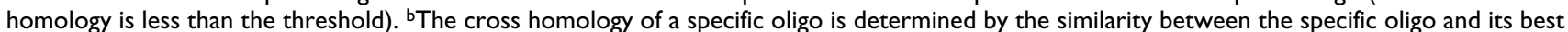
homology in the non-target sequence. CIAB, integration of artificial neural network (ANN) and basic local alignment search tool (BLAST). ${ }^{\mathrm{B}} \mathrm{BLAST}$ with $U_{d}$, BLAST search with the density of unique subsequences. $\mathrm{W}$ is word length in the parameters of BLAST.

unique markers in UMD. The IAB algorithm can effectively identify specific oligos that can serve as microarray probes, siRNAs, and primers. To demonstrate the specific oligo prediction ability of this algorithm, the wholegenome primer sets of $X c c$ strain 17 and human were designed and validated using biological PCR experiments. SpecificDB, derived from the IAB algorithm, is not only a comprehensive bioinformatic database but is also a useful web server, and is useful for functional genomics and systems biology studies.

\section{Methods}

\section{The construction of UMD}

The subsequences, with lengths between 10 and 26 nucleotides, of gene sequences in the database were identified by encoding. Figure 1a shows that a subsequence slides the window (one nucleotide at a time) along the TC sequence and a stack of subsequences is then collected. Every subsequence is encoded using the following formula:

Table 3: Performance comparison for 70-mer RGI.

\begin{tabular}{ccccc}
\hline Procedure & Sensitivity factor & rate (\%) & Cross homology & Execution time (hours) \\
\hline IABc for HGI $^{\mathrm{a}}$ & 0.2 & 99 & 0.53 & 0.09 \\
IAB for RGle & 0.3 & 99 & 0.53 & 0.11 \\
Pure BLAST & 0.2 & 99 & 0.53 & 0.10 \\
BLAST with $U_{d}^{f}$ & 0.3 & 99 & 0.53 & 0.12 \\
\end{tabular}

${ }^{a}$ The success rate is the percentage of tentative human consensus sequences on which the procedure can find the specific oligo (where the cross homology is less than the threshold). ${ }^{b}$ The cross homology of a specific oligo is determined by the similarity between the specific oligo and its best homology in the non-target sequence. $\mathrm{IIB}^{\mathrm{A}}$, integration of artificial neural network (ANN) and basic local alignment search tool (BLAST). ${ }^{\mathrm{H} H G I}$, human gene index.

eRGI, rat gene index. fBLAST with $U_{d}$, BLAST search with the density of unique subsequences. 


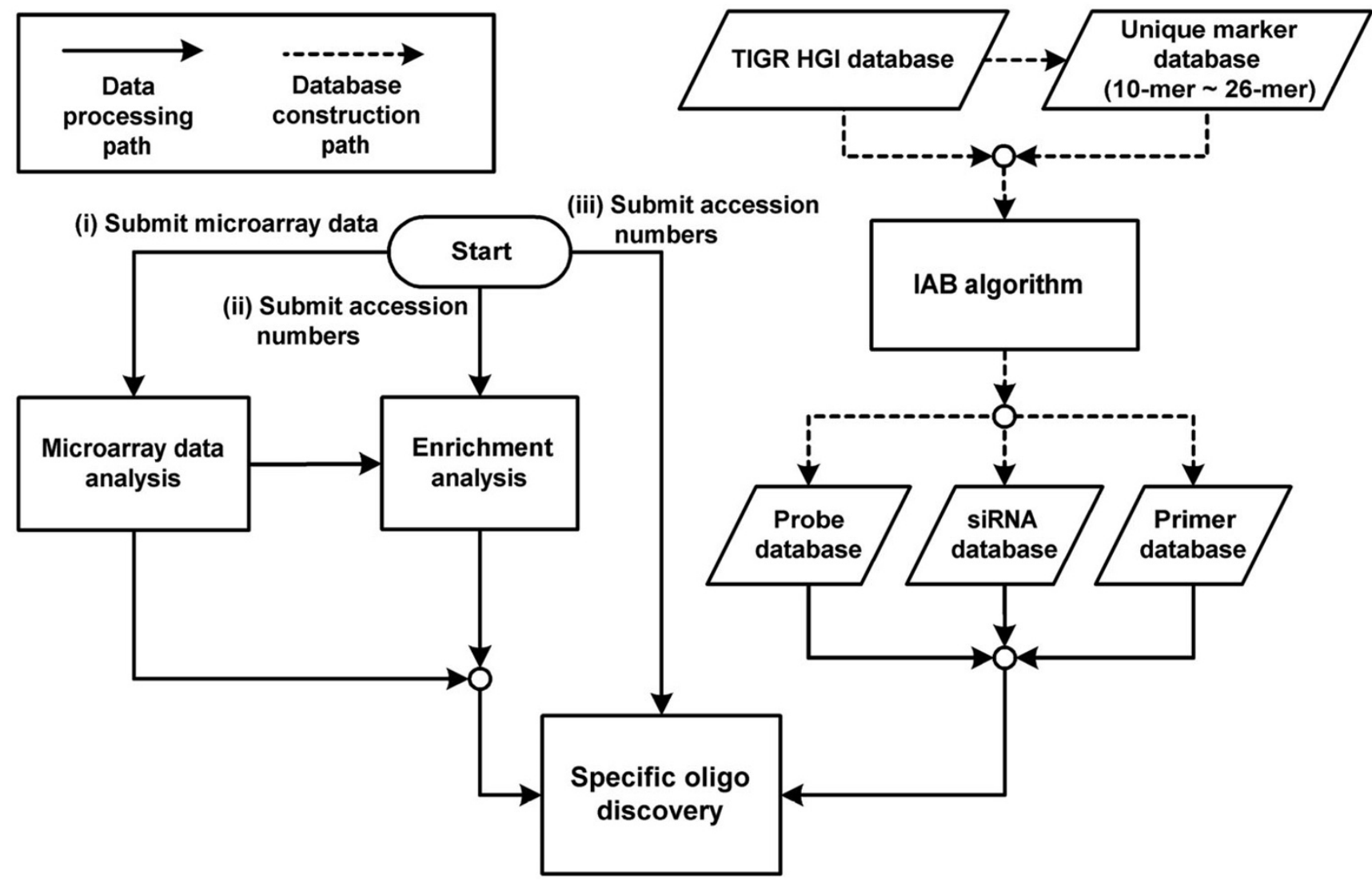

Figure 5

The architecture of SpecificDB. The web server SpecificDB includes the probe, siRNA, and primer databases, and integrates the IAB algorithm, microarray data analysis, and enrichment analysis. There are three initial workflows into which users can submit data: (i) microarray data can be submitted to the microarray data analysis component; (ii) GenBank accession numbers or UniGene IDs of a group of genes can be submitted to the enrichment analysis component; and (iii) GenBank accession numbers or UniGene IDs of a group of genes can be submitted to the specific oligo discovery component.

$$
\text { code }=\sum_{i=1}^{l} c_{i} \times 4^{i-1}
$$

Where $c_{i}$ is $0,1,2$, or 3 for A, C, G or T at the $i$-th base of the subsequence and $l$ is the length of the subsequence. For example, a sequence such as ACGTC has the coding number of $0 \times 4^{0}+1 \times 4^{1}+2 \times 4^{2}+3 \times 4^{3}+1 \times 4^{4}=484$ and $l=$ 5. Using this encoding formula, subsequences of different DNA sequences have different coding numbers.

We used the hash table algorithm to obtain the positions of all unique subsequences that were stored in UMD. A flowchart depicting the procedures is shown in Figure 2, which indicates that the subsequences for all of the genes in the database were encoded and placed in the hash table using the coding number of the subsequences as the hash key. Moreover, if the subsequence appears only once (frequency is one), then the subsequence is unique with at least one nucleotide mismatch to all of the other sequences in the entire sequence database.

We created 10-mer 26-mer marker tables and stored the locations of all unique subsequences with 10-mer 26mer for every THC sequence. The element of ANN's input vector is the density of unique subsequences $\left(U_{d}\right)$ of an oligo. The parameter $U_{d}$ is defined as follows:

$$
U_{d}=\frac{N_{\text {unique }}}{L}
$$

where $N_{\text {unique }}$ is the number of unique subsequence in the oligo (Figure 1a) and $L$ is the length of the oligo. According to the previous report [1], the larger value of $U_{d}$ indicates sequence specificity. Therefore, we used $U_{d}$ as the element of ANN's input vector. 

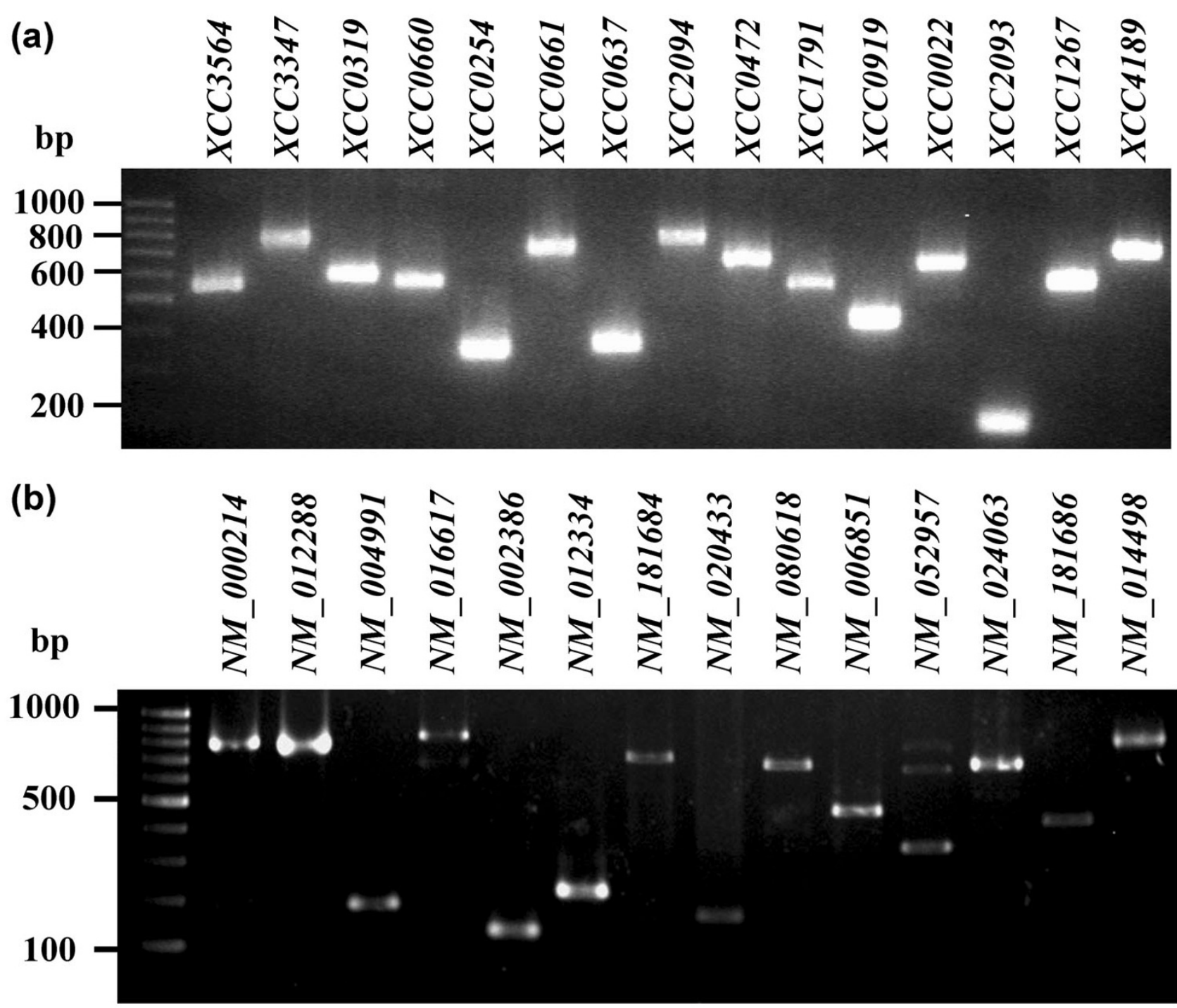

(c)

\begin{tabular}{|c|c|c|c|c|c|}
\hline & \multicolumn{3}{|c|}{$X c c$} & \multirow{2}{*}{$\begin{array}{l}\text { Human } \\
\text { Random } \\
\text { selection }\end{array}$} & \multirow[b]{2}{*}{$\begin{array}{l}\text { Total } \\
\text { genes }\end{array}$} \\
\hline & $\begin{array}{l}\text { rpoE } \\
\text { related }\end{array}$ & $\begin{array}{c}\text { sos } \\
\text { response }\end{array}$ & $\begin{array}{l}\text { Random } \\
\text { selection }\end{array}$ & & \\
\hline Gene no. & 18 & 29 & 49 & 15 & 111 \\
\hline Success no. & 16 & 26 & 49 & 14 & 105 \\
\hline Success rate & $89 \%$ & $90 \%$ & $100 \%$ & $93 \%$ & $95 \%$ \\
\hline
\end{tabular}

Figure 6

PCR amplification by using the primers predicted by the IAB algorithm. 96 Xcc and I5 human primer sets were selected for PCR amplification. (a) Electrophoretic analysis of Xcc PCR products. Representative results of PCR amplification for 15 genes are shown. (b) Electrophoretic analysis of human PCR products. Fourteen genes with correct size of PCR products are shown. (c) The success rates of PCR amplification for Xcc and human are presented. The total success rate was $95 \%$ 
Table 4: The integration of ANN and BLAST (IAB algorithm).

\begin{tabular}{|c|c|}
\hline $\mathbf{I A B}^{\mathrm{a}}(T, N, F)^{\mathrm{b}}$ & \\
\hline I. & size $\leftarrow$ length $[T]-N$ \\
\hline 2. & Oligo allOligo [size] $/ *$ the data structure Oligo includes score and sequence*/ \\
\hline 3. & for pos $\leftarrow 1$ to size \\
\hline 4. & do allOligo [pos].sequence $\leftarrow$ T.substring (pos, pos $+N$ ) \\
\hline 5. & I* calculate the ANN score for each sliding oligo using the trained ANN *I \\
\hline 6. & allOligo [pos].score = CalScoreByNN c (allOligo [pos ]) \\
\hline 7. & sort the allOligo array into non-decreasing order by ANN score \\
\hline 8. & lowestSim $\leftarrow 1.0$ \\
\hline 9. & for $p \leftarrow 0$ to $\left(\right.$ size $\left.\cdot F^{d}\right)$ \\
\hline 10. & do oligo $\leftarrow$ allOligo $[p]$.sequence \\
\hline 11. & similarity $\leftarrow$ CalSimByBlast $\mathrm{e}$ (oligo) \\
\hline 12. & if similarity $<$ lowestSim \\
\hline 13. & then bestOligo $\leftarrow$ oligo \\
\hline 14. & lowestSim $\leftarrow$ similarity \\
\hline 15. & if lowest Sim $<\mathrm{TH}_{\text {sim }}{ }^{\mathrm{f}}$ \\
\hline 16. & then return bestOligo \\
\hline 17. & return bestOligo \\
\hline
\end{tabular}

aIAB, integration of artificial neural network (ANN) and basic local alignment search tool (BLAST). bThe input parameters are as follows: the THC sequence $T$, the oligo length $N$, and the sensitivity factor $F$; ${ }^{c} \mathrm{CalScoreByNN}$ procedure calculates the ANN score (indicates the cross homology) by trained ANN; dThe sensitivity factor is set as $0.2,0.3$, and 0.4 ; ${ }^{\circ}$ CalSimByBlast procedure calculates the cross homology by WU-BLAST; fThe cross homology threshold $\left(\mathrm{TH}_{\text {sim }}\right)$ used for 70 -mer and 50 -mer was 0.7 and that used for 25 -mer was 0.8 .

Calculation of input vector for ANN from UMD

The input vector $\mathrm{X}$ is defined as follows:

$$
\mathrm{X}=\left\langle U_{d}^{(10-m e r)}, U_{d}^{(11-m e r)}, \ldots, U_{d}^{(26-m e r)}\right\rangle
$$

where $U_{d}^{(\mathrm{N}-m e r)}$ is the $U_{d}$ of $\mathrm{N}$-mer subsequence in an oligo. In Figure 1a, the solid triangles mark the starting position of the unique subsequences in an $\mathrm{N}$-mer oligo, and the count of the solid triangles is the number of unique subsequences. We then used the pre-established UMD to identify the location of a unique subsequence (solid triangle) and calculate $U_{d}$ immediately without searching the entire HGI or RGI database.

\section{Construction of data sets for training ANN}

We applied the previous calculation of input vector to create the training set from the HGI database, and a k-fold validation method was employed to improve the prediction performance [29]. Therefore, 10 original data sets were created according to the prefix, including the first 6 characters of the THC ID number, such as THC180 THC189.

All of the THC sequences with the same prefix were sorted out and put together as a data set. Then, we randomly selected 200 THC sequences from each original data set to create 10 data source (DS) sets, including $\mathrm{DS}_{\mathrm{THC} 180^{\prime}}$ $\mathrm{DS}_{\mathrm{THC181}}, \ldots, \mathrm{DS}_{\mathrm{THC189}}$. Each data source set contained 200 THC sequences with the same prefix. For example, the data source set $\mathrm{DS}_{\mathrm{THC} 181}$ contained 200 THC sequences randomly selected from THC1810000 to THC1819999. In this study, without losing generality, we chose $\mathrm{DS}_{\mathrm{THC} 180}$ as the test data source and the remainder $\left\{\mathrm{DS}_{\mathrm{THC181}}\right.$, $\left.\mathrm{DS}_{\mathrm{THC182}}, \ldots, \mathrm{DS}_{\mathrm{THC189}}\right\}$ as the training data source.

\section{Training set}

To construct the training set (TS), 100 70-mer oligos were randomly selected from each THC sequence from the training data source. Then, a total of nine training sets were derived from the corresponding DS, which were

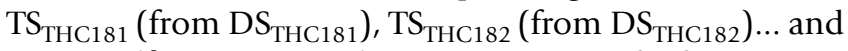
$\mathrm{TS}_{\mathrm{THC} 189}\left(\right.$ from $\mathrm{DS}_{\mathrm{THC} 189}$ ). Since every DS had $200 \mathrm{THC}$ sequences, it created a large training set containing 20,000 70 -mer oligos. The training execution time and prediction performance were considered. We then randomly selected 50 THC sequences from DS to produce 5,000 oligos in every TS.

\section{Test set}

To construct the test set, 100 70-mer oligos were randomly selected from each THC sequence of test data source $\mathrm{DS}_{\mathrm{THC} 180}$. Thus, the test set was an assembly of 20,000 oligos, because there were 200 THC sequences in $\mathrm{DS}_{\mathrm{THC} 180}$.

\section{Validation set}

In order to understand whether the prediction has generality on large-scale databases, we randomly selected two 70-mer oligos from every THC sequence in the entire HGI database, but skipped oligos with any base ambiguity symbol, to construct the validation set. The validation set 
had 389,146 oligos and covered $93.6 \%$ of the HGI database. The training set described above was subjected to various conditions such as number of hidden layer nodes, learning rate, and momentum, to obtain trained ANNs. The trained ANNs were further applied to both the test and validation sets and were checked to see if the results had the same trend. If the results of test and validation sets had inconsistent trends, these results were filtered out. Finally, we selected the ANN with the best performance from the trained ANNs with consistent trends for both the test and validation sets.

\section{ANN training}

The Java Object Oriented Neural Engine [45], an open source project that provides a highly adaptable ANN for Java programmers, was included in our programs. The training function we used was a batch-mode training algorithm and the training procedure was terminated when the number of iterations exceeded the maximum training epoch.

We employed the sigmoid activation function as both an input and output layer. The sigmoid function's output was smoothly limited within the range of 0 to 1 . The hidden layer was the logarithmic layer, which prevented the saturation of the processing elements of a layer under a lot of connected input synapses, or under input values very close to the limits 0 and 1 . The momentum was set to 0.5 and the learning rate was set to 0.1 in this study.

An ANN with one hidden layer was selected and the number of hidden layer nodes was determined based on the classification performance on training data. We trained four ANNs with different numbers of hidden layer nodes (4, 10, 16, and 22 nodes), and then selected the best ANN using RMSE [28]. The RMSE was defined as follows:

$$
\mathrm{RMSE}=\sqrt{\frac{\sum_{\mathrm{i}=1}^{\mathrm{n}}\left(y_{i}-\hat{\gamma}_{i}\right)^{2}}{n}}
$$

where $\mathrm{n}$ is the number of input vectors, $y_{i}$ is the output value of every input vector, and $\hat{y}_{i}$ is the desired output (from BLAST) of every input vector.

\section{Integration of ANN and BLAST (IAB algorithm)}

In this study, we designed an algorithm integrating ANN and BLAST (IAB algorithm) to identify specific N-mer oligos with high efficiency. The pseudo code of the IAB algorithm is shown in Table 4 and the architecture of IAB is shown in Figure 1. The cross homology of a specific oligo was determined by the similarity between the specific oligo and its best homology in the non-target sequences, and it was calculated by BLAST. The ANN score was the output value of the trained ANN and could indicate the cross homology.

A brief description of the IAB algorithm is as follows: (1) take one THC sequence as input and calculate the ANN score for each sliding $\mathrm{N}$-mer oligo of the input using the trained ANN (Lines $1 \sim 5$ ); (2) calculate the cross homology by WU-BLAST (oligo with the lowest ANN score is evaluated first) (Lines $6 \sim 15$ ); and (3) the procedure will be finished when the first specific oligo is found; otherwise, a certain percentage (sensitivity factor) of the oligos will be screened.

The sensitivity factor was defined as the maximum percentage of sliding oligos in the input gene sequence that would be screened by BLAST. In this study, we randomly selected 100 THC sequences on which to perform our algorithm. The cross homology threshold used for the 70mer and 50 -mer was $70 \%$ while that for 25 -mer was $80 \%$.

To investigate the performance with and without ANN under the same conditions (e.g. the length of oligos, test set, and genome), we carried out BLAST search and compared it with the results derived from the IAB algorithm. The procedures for pure BLAST search are as follows: (1) for each sliding N-mer oligo of the input THC sequence, the cross homology is calculated by WU-BLAST; (2) if the cross homology of any oligo is less than the threshold (i.e. the specific oligo), the first specific oligo is found and the procedure is finished; (3) if the procedure cannot find any specific oligo, it will screen all sliding $\mathrm{N}$-mer oligos and then return the oligo with the lowest cross homology. Pure BLAST is similar to the IAB algorithm (Table 4) but skips the calculation of the ANN scores. Although calculation of the ANN scores requires extra execution time, it could save more execution time by decreasing the number of BLAST calculations needed because the ANN score can help filter out non-specific oligos. Furthermore, in order to understand the performance of BLAST search with $U_{d^{\prime}}$ we have implemented a program that can sort the oligo candidates based on the summation of 10-mer 26-mer $U_{d}$ and BLAST each oligo candidate on the sorted list until a suitably specific oligo has been found.

\section{Probe design procedure}

The rules described by Chang and Peck [1] for probe selection were adopted in this study. Under the selection rules, sequence sections were discarded if they met any of the following criteria: (a) number of any single bases (As, Cs, Ts or Gs) exceeded half of the section length; (b) the length of any contiguous As, Cs, Ts, or Gs exceeded a quarter of the section length; (c) GC content was under $40 \%$ or over $60 \%$; or (d) no self-complementary region within the 
sequence section. The sequence sections that do not meet the above criteria are considered as candidate probes for further selection by our IAB algorithm. The ANN predicted the 10 most specific oligos for each THC, for which we then used WU-BLAST to calculate the cross homology. Finally, we filtered the oligos having high cross homology and displayed the top four probes.

\section{siRNA design procedure}

We used the UMD to get 19-mer unique markers for each THC sequence. Suppose that there were N unique oligos of 19-mer in a THC sequence, the ANN scores of these $\mathrm{N}$ oligos were calculated, and the N/3 oligos with the lowest ANN scores were selected because ANN scores indicate cross homology. Then we used the eight criteria described by Reynolds et al. to compute siRNA score [35]. We selected the top two oligos by the siRNA scores to calculate cross homology by WU-BLAST. Finally, we chose the best oligo using the cross homology of each oligo.

\section{Primer design procedure}

The primer design procedure was as follows: (a) used primer3 program [46] to produce primer candidates; (b) calculated the ANN score for all candidates; (c) selected top 10 primers with the highest ANN score to calculate the cross homology by WU-BLAST; and (d) the primer with the lowest cross homology was selected.

\section{The polymerase chain reaction for Xcc primer sets}

The PCR amplifications were carried out in a $50 \mu \mathrm{l}$ reaction mixture containing $1 \mathrm{X}$ buffer, $200 \mu \mathrm{M}$ dNTP, $0.2 \mu \mathrm{M}$ of each primer, $2 \mu \mathrm{l}$ DMSO, $200 \mathrm{ng}$ genomic DNA templates, and 2 units of thermostable polymerase (proTag plus; Protech Technology Enterprise Co., Taiwan). The PCR conditions were as follows: the 1 st cycle, $94^{\circ} \mathrm{C}$ for 5 $\min , 60^{\circ} \mathrm{C}$ for $1 \mathrm{~min}$ then $72^{\circ} \mathrm{C}$ for $1 \mathrm{~min}$; the $2^{\text {nd }}-30^{\text {th }}$ cycle: $94^{\circ} \mathrm{C}$ for $1 \mathrm{~min}, 60^{\circ} \mathrm{C}$ for $1 \mathrm{~min}$ then $72^{\circ} \mathrm{C}$ for 1 min, and a final extension for $10 \mathrm{~min}$ at $72^{\circ} \mathrm{C}$. The PCR products were then subjected to $1 \%$ of agarose gel electrophoresis.

\section{The polymerase chain reaction for human primer sets} The PCR amplifications were carried out in a $20 \mu \mathrm{l}$ reaction mixture containing 1X GI buffer (Takara), $200 \mu \mathrm{M}$ $\mathrm{dNTP}, 0.2 \mu \mathrm{M}$ of each primer, $100 \mathrm{ng}$ cDNA templates of lung cancer CL1-0 cell line, and 1 units of thermostable polymerase (proTag plus; Protech Technology Enterprise Co., Taiwan). The PCR conditions were as follows: the 1 st cycle, $94^{\circ} \mathrm{C}$ for $5 \mathrm{~min}$; the $2 \mathrm{nd}-30$ th cycle: $94^{\circ} \mathrm{C}$ for 1 $\min , 58^{\circ} \mathrm{C}$ for $1 \mathrm{~min}$ then $72^{\circ} \mathrm{C}$ for $1 \mathrm{~min}$, and a final extension for $10 \mathrm{~min}$ at $72^{\circ} \mathrm{C}$. The PCR products were then subjected to $2 \%$ of agarose gel electrophoresis.

\section{Abbreviations}

ANN: artificial neural network; BLAST: basic local alignment search tool; DS: data source; GO: gene ontology; HGI: human gene index; IAB: integration of ANN and BLAST; oligo: oligonucleotide; PCR: polymerase chain reaction; RGI: rat gene index; RMSE: root mean square error; siRNA: small interfering RNA; SpecificDB: specific oligo web server; TC: tentative consensus; THC: tentative human consensus; TIGR: the Institute for Genomic Research; TS: training set; $U_{d}$ : the density of unique subsequences; UMD: unique marker database; Xcc: Xanthomonas campestris pv. Campestris.

\section{Authors' contributions}

CCL, PCC, and JJWC conceived and designed the methods. CCL wrote the software program. CCL, KCL, PCY, and JJWC wrote the paper. CCL and WSEC conceived and designed the computational analysis. CCL, JCC and MTY designed and performed the biological experiments.

\section{Additional material}

\section{Additional file 1}

Excel spreadsheet, Supplemental Table S1. The genome-wide primer sets for the Xcc strain 17.

Click here for file

[http://www.biomedcentral.com/content/supplementary/14712105-8-164-S1.xls]

\section{Acknowledgements}

This work was supported by the National Chung-Hsing University Biotechnology Center through the Teaching Core Facility Project grant, as well as partially supported by the National Science Council grant (NSC 95-23 I 4-B005-005-MY3). We are grateful to Mrs. Karri Aston for copyediting the manuscript.

\section{References}

I. Chang PC, Peck K: Design and assessment of a fast algorithm for identifying specific probes for human and mouse genes. Bioinformatics 2003, I9(II): 1311-1317.

2. Chen JJ, Peck K, Hong TM, Yang SC, Sher YP, Shih JY, Wu R, Cheng JL, Roffler SR, Wu CW, Yang PC: Global analysis of gene expression in invasion by a lung cancer model. Cancer Res 200I, 6I(13):5223-5230.

3. Chen JJ, Lin YC, Yao PL, Yuan A, Chen HY, Shun CT, Tsai MF, Chen $\mathrm{CH}$, Yang PC: Tumor-associated macrophages: the doubleedged sword in cancer progression. J Clin Oncol 2005, 23(5): 953-964.

4. Liu CC, Chen WS, Lin CC, Liu HC, Chen HY, Yang PC, Chang PC, Chen J]: Topology-based cancer classification and related pathway mining using microarray data. Nucleic Acids Res 2006, 34(14):4069-4080.

5. Evertsz EM, Au-Young J, Ruvolo MV, Lim AC, Reynolds MA: Hybridization cross-reactivity within homologous gene families on glass cDNA microarrays. Biotechniques 200।, 3 I(5): I I82, I I84, II 86 passim.

6. Modrek B, Lee C: A genomic view of alternative splicing. Nat Genet 2002, 30(I):13-19.

7. Xu W, Bak S, Decker A, Paquette SM, Feyereisen R, Galbraith DW: Microarray-based analysis of gene expression in very large 
gene families: the cytochrome P450 gene superfamily of Arabidopsis thaliana. Gene 200I, 272(I-2):6I-74.

8. Nielsen HB, Knudsen S: Avoiding cross hybridization by choosing nonredundant targets on cDNA arrays. Bioinformatics 2002, I 8(2):32I-322.

9. Yuan B, Latek R, Hossbach M, Tuschl T, Lewitter F: siRNA Selection Server: an automated siRNA oligonucleotide prediction server. Nucleic Acids Res 2004, 32(Web Server issue):WI30-4.

10. Cuticchia AJ, Arnold J, Timberlake WE: PCAP: probe choice and analysis package--a set of programs to aid in choosing synthetic oligomers for contig mapping. Comput Appl Biosci 1993, 9(2):20I-203.

1I. Fu YX, Timberlake WE, Arnold J: On the design of genome mapping experiments using short synthetic oligonucleotides. Biometrics 1992, 48(2):337-359.

12. Herwig R, Schmitt AO, Steinfath M, O'Brien J, Seidel H, Meier-Ewert $S$, Lehrach H, Radelof U: Information theoretical probe selection for hybridisation experiments. Bioinformatics 2000, I 6(10):890-898.

13. Li F, Stormo GD: Selection of optimal DNA oligos for gene expression arrays. Bioinformatics 200I, 17(II):1067-1076.

14. Kaderali L, Schliep A: Selecting signature oligonucleotides to identify organisms using DNA arrays. Bioinformatics 2002, I 8(10): I340-1349.

15. Rouillard JM, Herbert CJ, Zuker M: OligoArray: genome-scale oligonucleotide design for microarrays. Bioinformatics 2002, I 8(3):486-487.

16. Sung WK, Lee WH: Fast and accurate probe selection algorithm for large genomes. Proc IEEE Comput Soc Bioinform Conf 2003, 2:65-74.

17. Hyyro H, Juhola M, Vihinen M: Genome-wide selection of unique and valid oligonucleotides. Nucleic Acids Res 2005, 33(I3):el I 5.

18. Baldi PF, Brunak S: Bioinformatics: The machine learning approach. Cambridge, MA, MIT Press; 1998:91-104.

19. Chalk AM, Sonnhammer EL: Computational antisense oligo prediction with a neural network model. Bioinformatics 2002 I 8(I 2): I567-I575.

20. Shabalina SA, Spiridonov AN, Ogurtsov AY: Computational models with thermodynamic and composition features improve siRNA design. BMC Bioinformatics 2006, 7:65.

21. Lisboa PJ, Taktak AF: The use of artificial neural networks in decision support in cancer: a systematic review. Neural Netw 2006, I (9): 4 :408-4I5

22. Han L, Cui J, Lin H, Ji Z, Cao Z, Li Y, Chen Y: Recent progresses in the application of machine learning approach for predicting protein functional class independent of sequence similarity. Proteomics 2006, 6(14):4023-4037.

23. Wu C, Whitson G, McLarty J, Ermongkonchai A, Chang TC: Protein classification artificial neural system. Protein Sci 1992, I(5):667-677.

24. Linder R, Dew D, Sudhoff H, Theegarten D, Remberger K, Poppl S, Wagner M: The 'subsequent artificial neural network' (SANN) approach might bring more classificatory power to ANNbased DNA microarray analyses. Bioinformatics 2004, 20(I8):3544-3552.

25. Quackenbush J, Cho J, Lee D, Liang F, Holt I, Karamycheva S, Parvizi B, Pertea G, Sultana R, White J: The TIGR Gene Indices: analysis of gene transcript sequences in highly sampled eukaryotic species. Nucleic Acids Res 200 I, 29(I): 159-164.

26. WU-BLAST [http://blast.wustl.edu/]

27. Lawrence S, Giles CL, Tsoi AC: Lessons in Neural Network Training: overfitting may be harder than expected. In Proceedings of the Fourteenth National Conference Artificial Intelligence, AAAl-97 AAAI Press; 1997.

28. Freund JE: Modern elementary statistic. Pearson Education, Inc.; 2004.

29. Yu JS, Ongarello S, Fiedler R, Chen XW, Toffolo G, Cobelli C, Trajanoski Z: Ovarian cancer identification based on dimensionality reduction for high-throughput mass spectrometry data. Bioinformatics 2005, 2 I ( I 0):2200-2209.

30. Liu CC, Lin CC, Chen WS, Chen HY, Chang PC, Chen JJ, Yang PC: CRSD: a comprehensive web server for composite regulatory signature discovery. Nucleic Acids Res 2006, 34(Web Server issue):W57|-7.

31. SpecificDB [http://biochip.nchu.edu.tw/SpecificDB/]
32. da Silva AC, Ferro JA, Reinach FC, Farah CS, Furlan LR, Quaggio RB, Monteiro-Vitorello CB, Van Sluys MA, Almeida NF, Alves LM, do Amaral AM, Bertolini MC, Camargo LE, Camarotte G, Cannavan F, Cardozo J, Chambergo F, Ciapina LP, Cicarelli RM, Coutinho LL, Cursino-Santos JR, El-Dorry H, Faria JB, Ferreira AJ, Ferreira RC, Ferro MI, Formighieri EF, Franco MC, Greggio CC, Gruber A, Katsuyama AM, Kishi LT, Leite RP, Lemos EG, Lemos MV, Locali EC, Machado MA, Madeira AM, Martinez-Rossi NM, Martins EC, Meidanis J, Menck CF, Miyaki CY, Moon DH, Moreira LM, Novo MT, Okura VK, Oliveira MC, Oliveira VR, Pereira HA, Rossi A, Sena JA, Silva C, de Souza RF, Spinola LA, Takita MA, Tamura RE, Teixeira EC, Tezza RI, Trindade dos Santos M, Truffi D, Tsai SM, White FF, Setubal JC, Kitajima JP: Comparison of the genomes of two Xanthomonas pathogens with differing host specificities. Nature 2002, 4I 7(6887):459-463.

33. Little JW, Mount DW: The SOS regulatory system of Escherichia coli. Cell 1982, 29(I):II-22.

34. Rouviere PE, De Las Penas A, Mecsas J, Lu CZ, Rudd KE, Gross CA rpoE, the gene encoding the second heat-shock sigma factor, sigma E, in Escherichia coli. Embo J 1995, I4(5): 1032-1042.

35. Reynolds A, Leake D, Boese Q, Scaringe S, Marshall WS, Khvorova A Rational siRNA design for RNA interference. Nat Biotechnol 2004, 22(3):326-330.

36. Wang $X$, Seed B: Selection of oligonucleotide probes for protein coding sequences. Bioinformatics 2003, I9(7):796-802.

37. Matveeva OV, Shabalina SA, Nemtsov VA, Tsodikov AD, Gesteland RF, Atkins JF: Thermodynamic calculations and statistical correlations for oligo-probes design. Nucleic Acids Res 2003, 3 I ( 14):42II-42I7.

38. Bernaola-Galvan P, Roman-Roldan R, Oliver JL: Compositional segmentation and long-range fractal correlations in DNA sequences. Physical Review E Statistical Physics, Plasmas, Fluids, and Related Interdisciplinary Topics 1996, 53(5):5181-5189.

39. Snove $\mathrm{O}$ Jr., Holen T: Many commonly used siRNAs risk off-target activity. Biochem Biophys Res Commun 2004, 31 9(I):256-263.

40. Yamada T, Morishita S: Accelerated off-target search algorithm for siRNA. Bioinformatics 2005, 2 | (8):|3|6-|324.

4I. Benitez JM, Castro JL, Requena I: Are artificial neural networks black boxes? Neural Networks, IEEE Transactions on 1997, 8(5): I I56-II64.

42. Huang YC, Chang CF, Chan CH, Yeh TJ, Chang YC, Chen CC, Kao $\mathrm{CY}$ : Integrated minimum-set primers and unique probe design algorithms for differential detection on symptomrelated pathogens. Bioinformatics 2005, 2 I (24):4330-4337.

43. Cobo F, Stacey GN, Hunt C, Cabrera C, Nieto A, Montes R, Cortes JL, Catalina P, Barnie A, Concha A: Microbiological control in stem cell banks: approaches to standardisation. Appl Microbiol Biotechnol 2005, 68(4):456-466.

44. Kane MD, Jatkoe TA, Stumpf CR, Lu J, Thomas JD, Madore SJ: Assessment of the sensitivity and specificity of oligonucleotide (50mer) microarrays. Nucleic Acids Res 2000, 28(22):4552-4557.

45. Java Object Oriented Neural Engine [http://www.joone world.com/]

46. Rozen S, Skaletsky H: Primer3 on the WWW for general users and for biologist programmers. Methods Mol Biol 2000, 132:365-386.

Publish with Bio Med Central and every scientist can read your work free of charge

"BioMed Central will be the most significant development for disseminating the results of biomedical research in our lifetime. "

Sir Paul Nurse, Cancer Research UK

Your research papers will be:

- available free of charge to the entire biomedical community

- peer reviewed and published immediately upon acceptance

- cited in PubMed and archived on PubMed Central

- yours - you keep the copyright
BioMedcentral 